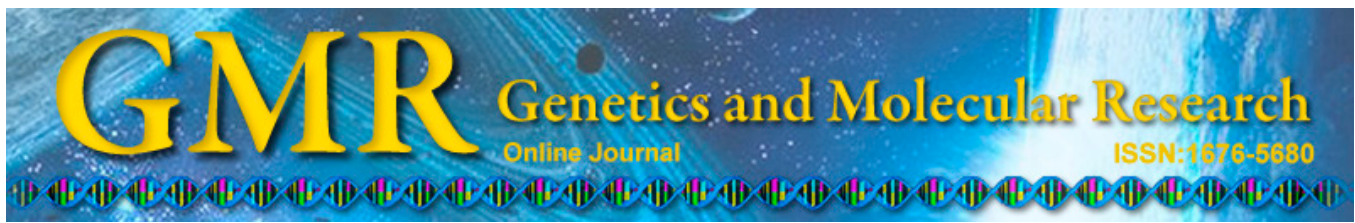

\title{
Characterization and expression analysis of somatic embryogenesis receptor-like kinase genes from Phalaenopsis
}

\author{
Y.W. Huang ${ }^{1}$, Y.J. Tsai ${ }^{2}$ and F.C. Chen ${ }^{1,2}$ \\ ${ }^{1}$ Graduate Institute of Plant Science, \\ National Pingtung University of Science and Technology, Pingtung, Taiwan \\ ${ }^{2}$ Department of Plant Industry, \\ National Pingtung University of Science and Technology, Pingtung, Taiwan \\ Corresponding author: F.C. Chen \\ E-mail: fchen@mail.npust.edu.tw
}

Genet. Mol. Res. 13 (4): 10690-10703 (2014)

Received October 21, 2013

Accepted January 13, 2014

Published December 18, 2014

DOI http://dx.doi.org/10.4238/2014.December.18.11

\begin{abstract}
Somatic embryogenesis receptor-like kinase (SERK) genes have been found to be involved in the somatic embryogenesis of several plant species. We identified and characterized 5 PhSERK genes in the Phalaenopsis orchid. The amino acid sequences of PhSERKs and other SERK proteins are highly conserved, with the highest homology observed in the leucine-rich repeat-receptor-like kinase domain. All 5 PhSERKs were expressed in all Phalaenopsis organs examined (root, leaf, shoot apical meristem, and flower), with the strongest expression, particularly for PhSERK1 and 3, in the shoot apical meristem of mature plants. Expression of all PhSERKs was downregulated during early floral bud development and was upregulated gradually until the semiopen flower stage was reached. All 5 PhSERKs were expressed during both seed germination and protocorm-like-body (PLB) development. In germinated seeds, quantitative real-time PCR revealed upregulation of all PhSERKs except PhSERK4 at 1 week and downregulation after 4 weeks. The 5 PhSERKs were differentially expressed in the early stage
\end{abstract}


of PLB development and maintained substantial levels during PLB formation, with PhSERK1 and 5 upregulated 1 week after culture and PhSERK2, 3, and 4 downregulated over this period. Because physical wounding of PLB stimulates secondary PLB formation, the PhSERK5 expression peak at week 3 coincided with visible and fully developed secondary PLBs. PhSERK5 may be important in PLB induction and subsequent development. Our PhSERK expression analysis revealed that these genes have a broad role during orchid plant development.

Key words: Phalaenopsis; Leucine-rich repeat-receptor-like kinases; SERK; Protocorm-like body

\section{INTRODUCTION}

Somatic embryogenesis is a cellular differentiation pathway that converts totipotent embryogenic cells into somatic embryos during plant tissue culture and is important for plant propagation. To better manipulate plant regeneration via somatic embryogenesis, it is important to understand the molecular events underlying the transition from somatic to embryogenic cells (Yang et al., 2011). Both biochemical and morphological changes occur throughout the development of induced tissues during somatic embryogenesis (Santos et al., 2005). Genes involved in somatic embryogenesis include somatic embryogenesis receptor-like kinase (SERK; Schmidt et al., 1997), leafy cotyledon (Ledwon and Gaj, 2009, 2011), agamous-like 15 (Thakare et al., 2008), baby boom (Boutilier et al., 2002), and wuschel (Su et al., 2009).

The SERK gene is thought to play an important role in somatic embryogenesis (Schmidt et al., 1997; Sharma et al., 2008; Singla et al., 2008). It encodes a protein that belongs to the receptor-like kinase (RLK) family, which is highly conserved among plant species. It carries an external leucine-rich repeat (LRR) domain that is typically found in this class of protein (Santos et al., 2005). SERK genes play important roles in the response to external stimuli, cell-cell recognition during development, defense against pathogens, and self-incompatibility (Shiu and Bleecker, 2001). The first SERK gene, identified from carrot (DcSERK), was specifically expressed in competent cells during the globular stage (Schmidt et al., 1997). DcSERK is most closely related to AtSERK1 in Arabidopsis, which is expressed in embryogenic cells and during early somatic embryogenesis (Hecht et al., 2001). Indeed, transgenic Arabidopsis plants overexpressing AtSERK1 showed an enhanced capacity to initiate somatic embryogenesis during in vitro culture (Hecht et al., 2001). Moreover, AtSERK1 and 2 play a role in male sporogenesis, particularly tapetum development (Albrecht et al., 2005; Colcombet et al., 2005). ZmSERK1 and 2 of maize are similar to carrot and Arabidopsis SERK genes and are expressed in embryogenic calli as well as male and female reproductive tissues (Baudino et al., 2001). OsSERK1 is thought to be partially responsible for mediating defense signal transduction and has a basic role in rice somatic embryogenesis (Hu et al., 2005). More homologs of SERK have been identified in several plant species, including Dactylis glomerata (Somleva et al., 2000), Medicago truncatula (Nolan et al., 2003, 2009), Ocotea catharinensis (Santa-Catarina et al., 2004), Helianthus annuus (Thomas et al., 2004), Theobroma cacao (Santos et al., 2005), Citrus unshiu (Shimada et al., 2005), Triticum aestivum (Singla et al., 2008), Vitis vinifera (Schellenbaum et al., 2008), Solanum tuberosum (Sharma et al., 2008), Cocos nucifera (Perez-Núñez et al., 2009), Lactuca sativa (Santos et al., 2009), Rosa hybrida 
(Zakizadeh et al., 2010), Musa acuminata (Huang et al., 2010), Secale cereale (Gruszczyńska and Rakoczy-Trojanowska, 2011), Glycine max (Yang et al., 2011), Cyclamen persicum (Savona et al., 2012), Ananas comosus (Ma et al., 2012a,b), and Cyrtochilum loxense (Cueva et al., 2012). These reports all point to SERK as a specific marker of embryogenic competence during morphogenesis induction.

Phalaenopsis is one of the most important orchids for potted plant production in the global flower market. Mass production of orchids is achieved by in vitro germination of seeds, which develop into a structure called a protocorm and then into seedlings, or by induction of protocorm-like bodies (PLBs), which are equivalent to somatic embryos, and subsequent plantlet regeneration (Ishii et al., 1998; Mayer et al., 2010). A number of studies examining Phalaenopsis PLB induction and multiplication used explant sources, including flower stalk nodes and internodes (Reisinger et al., 1976; Lin, 1986), PLB sections (Young et al., 2000), leaves (Park et al., 2002), shoot tips (Tokuhara and Mii, 1993), and root tips (Park et al., 2003).

The developmental process of PLB formation and the underlying molecular events have not been explored in Phalaenopsis. Information regarding the function of SERK in regulating somatic embryogenesis in other plant species led us to investigate the contribution of PhSERK genes to PLB formation in Phalaenopsis by temporal and spatial expression analysis of protocorms and PLBs, in addition to their expression profile in various tissues. We found that the expressions of some PhSERK transcripts were related to cellular totipotency during in vitro development of PLBs of this orchid.

\section{MATERIAL AND METHODS}

\section{Plant material and explant preparation}

Potted plants of a hybrid, Phalaenopsis Sogo Vivien 'F858', were grown in the fanand-pad greenhouse of National Pingtung University of Science and Technology (Pingtung, Taiwan) under $70 \%$ shade light and controlled temperatures ranging from $27^{\circ}-30^{\circ} \mathrm{C}$. Total RNA was extracted from root tip, leaf, shoot apical meristem (SAM), and inflorescence for real-time polymerase chain reaction (PCR) of the expression of PhSERKs in mature plants. Total RNA was extracted from floral buds of different sizes to analyze relevant development stages: buds of 0.2-0.29 cm (FB1), 0.3-0.39 cm (FB2), 0.4-0.49 cm (FB3), 0.5-0.59 cm (FB4), $0.6-0.79 \mathrm{~cm}$ (FB5), and 0.8-0.99 cm (FB6); semi-open flowers (FB7); and fully open flowers (FB8). RNA was also extracted from different floral parts of the fully open flower, including the sepal, petal, labellum, column, and pollinia (Figure S1).

Protocorms of Phalaenopsis were obtained from in vitro-germinated seeds. The seeds were surface-sterilized with $0.6 \%$ sodium hypochlorite for $10 \mathrm{~min}$ and rinsed with sterile water 3 times. Seeds were sown on germination medium of (in g/L) 3 Hyponex $\left(7 \mathrm{~N}-6 \mathrm{P}_{2} \mathrm{O}_{5}-\right.$ $19 \mathrm{~K}_{2} \mathrm{O}$ ), 1 bacto-peptone, 50 potato homogenate, 50 banana paste, 30 sucrose, 2 activated charcoal, and 8 agar (Sigma-Aldrich. St. Louis, MO, USA). The medium $\mathrm{pH}$ was adjusted to 5.6 before autoclave sterilization and pouring into Petri dishes.

PLBs were induced by culture of in vitro leaf segments on 1/4 MS medium, which includes 1/4-strength macro- and micro-elements of MS medium (Murashige and Skoog, 1962) supplemented with $0.5 \mathrm{mg} / \mathrm{L}$ thidiazuron and $7 \mathrm{~g} / \mathrm{L}$ agar (Sigma-Aldrich), and $30 \mathrm{~g} / \mathrm{L}$ sucrose according to the method described by Hsu and Chen (2003). The basal half of primary PLBs was used for explants after removing the bisectioned apical top and was incubated on induc- 
tion medium consisting of (in $\left.\mathrm{g} \mathrm{l}^{-1}\right) 3$ Hyponex fertilizer $\left(7 \mathrm{~N}-6 \mathrm{P}_{2} \mathrm{O}_{5}-19 \mathrm{~K}_{2} \mathrm{O}\right), 1$ bacto-tryptone, 50 potato homogenate, 50 banana homogenate, 30 sucrose, 2 activated charcoal, and 8 agar (Sigma-Aldrich). The medium was prepared as described above. Explants were incubated in the dark at $25^{\circ} \pm 2{ }^{\circ} \mathrm{C}$, and 10 explants were included in each Petri dish as a replicate, with 4 replications for each cutting treatment. Primary PLBs developed from leaf sections after 4 months were used as explants for subsequent experiments.

The following stages of protocorms and PLBs were used for real-time PCR analysis: P0 (mature dried seeds before transfer onto germination medium; P1, P2, P3, P4, P5, and P6 (protocorms developed from seeds by germination for 1, 2, 3, 4, 5, 8 weeks, respectively; PLB0-5 (the basal half of primary PLBs on induction medium for 0 to 5 weeks, respectively) (Figure 1).

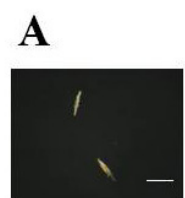

P0

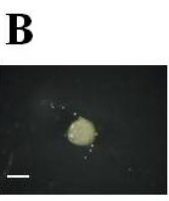

PLB0

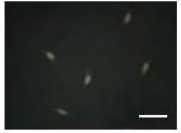

P1

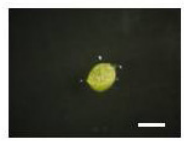

PLB1

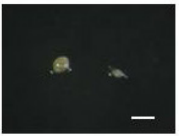

P2

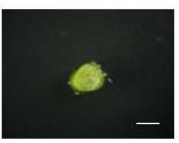

PLB2

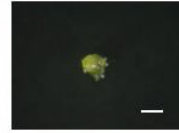

P3

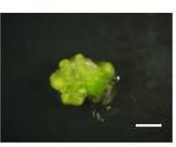

PLB3

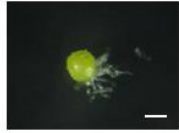

P4

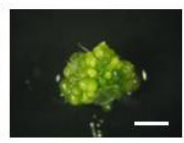

PLB4

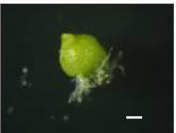

P5

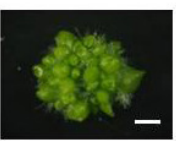

PLB5

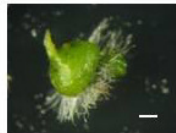

P6

weeks

0 1 2 3 4 5 8

Figure 1. Protocorm development (A) and protocorm-like body (PLB) formation (B) in Phalaenopsis. P0-P6, seeds germinated into protocorm for $0,1,2,3,4,5$, and 8 weeks, respectively. PLB0-5, basal parts of primary PLB after cutting and growth on induction medium for $0,1,2,3,4$, and 5 weeks, respectively. P0 (bar = 0.1 cm), P1-6 and PLB0-5 (bar $=0.2 \mathrm{~cm})$.

\section{RNA extraction and cDNA synthesis}

Total RNA was isolated and purified using the CTAB reagent as described (Chang et al., 1993) with modification. RNA pellets were resuspended in DEPC-treated sterile water and then precipitated with $0.3 \mathrm{X}$ volumes $8 \mathrm{M} \mathrm{LiCl}$. The total RNA solution was treated with RNase-free DNase (Promega, Madison, WI, USA) at $37^{\circ} \mathrm{C}$ for $30 \mathrm{~min}$ to remove residual genomic DNA. Purified RNA was quantified using a spectrophotometer (U2900, Hitachi, Tokyo, Japan), and aliquots were separated on a $0.8 \%$ agarose gel in $0.8 \mathrm{X}$ TE buffer to evaluate sample quality. Next, $2 \mu \mathrm{L}$ RNA was used to synthesize single-stranded cDNA using the SMART cDNA PCR Synthesis Kit (Clontech, Mountain View, CA, USA). Synthesized sscDNA fragments were used as templates for real-time PCR. 


\section{Isolation of PhSERKS}

Degenerate primers (Table S1) designed from SERK conserved domains were used to clone portion of the cDNA sequence. These primers were used for PCR amplification with cDNA from tight flower buds of Phalaenopsis. The amplified fragment was cloned into the pGEM-T Easy vector (Promega) and sequenced. Gene-specific primers (Table S1) were designed based on the known sequence of the PhSERK1 fragment for rapid amplification of $5^{\prime}$ and $3^{\prime}$ cDNA ends (RACE) using the SMART RACE cDNA amplification Kit (Clontech). The 5'-RACE and 3'-RACE products were cloned into the pGEM-T Easy vector and sequenced. Based on RACE sequence data, the PhSERK1-F and PhSERK1-R primers (Table S1) were designed to amplify full-length cDNA using Ex Taq (Takara, Shiga, Japan) and designated PhSERK1. Other PhSERK sequences were retrieved from Orchidstra (http:// orchidstra.abrc.sinica.edu.tw/none/) as partial expressed sequence tags ( $\mathrm{Su}$ et al., 2009), with redundant sequences removed after multiple alignments using ClustalW2 (http:// www.ebi.ac.uk/Tools/msa/clustalw2/), and were designated PhSERK2, 3, 4, and 5. Specific primers were designed to amplify non-conserved regions of each gene using PCR for the following specific DNA fragments: PhSERK2 (PATC125548), PhSERK3 (PAVEGC012987), PhSERK4 (PAALLC028299), and PhSERK5 (PATC125663) (Table S1). To obtain the fulllength for cDNA of PhSERK2 to 5, the same RACE strategy was adopted as for PhSERK1 using specific primer sequences (Table S1).

\section{Characterization of PhSERKs and phylogenetic analysis}

The signal peptide was predicted using the SignalP 4.0 Server tool (http://www.cbs. dtu.dk/services/SignalP/). The PSORT (http://psort.hgc.jp/) was used for protein prediction. To understand the phylogenetic relationship between PhSERK and known SERK genes of other plant species, multiple alignment was performed using Molecular Evolutionary Genetics Analysis (MEGA) 4.0 (Tamura et al. 2007). Sequence data were analyzed by neighbor-joining with the NEIGHBOR program in Phylogeny Inference Package (PHYLIP) and Unweighted Pair Group Mean Average methods in MEGA. Distances were calculated using DNADIST in PHYLIP. Bootstrapping and decay analysis involved neighbor-joining plots. Parsimony analysis and determination of clades involved use of MEGA.

\section{Quantification of transcript levels by real-time PCR assay}

Quantitative real-time PCR and data analysis involved the ABI PRISM 7300 Sequence Detection System (Applied Biosystems, Foster City, CA, USA) with SYBR Green PCR Master Mix (Applied Biosystems). Total RNA was isolated from Phalaenopsis tissues, and $2 \mu \mathrm{L}$ was used for first-strand cDNA synthesis by priming with $10 \mu \mathrm{M}$ oligo $(\mathrm{dT})_{12-18}$ Primer (Invitrogen, Carlsbad, CA, USA) and reverse transcribed with the SuperScript first-strand synthesis system (Invitrogen). Specific primers used for PhSERK gene expression analysis (Table 1) were designed to amplify specific regions of individual genes. The amplification program was $95^{\circ} \mathrm{C}$ for $10 \mathrm{~min}, 1$ cycle; $95^{\circ} \mathrm{C}$ for $15 \mathrm{~s}, 60^{\circ} \mathrm{C}$ for $1 \mathrm{~min}, 40$ cycles. The PhActin housekeeping gene was used as an internal control (Table 1). The optimal concentrations of target genes and the internal control PhActin were determined according to Chen et al. (2005). 
Three replicates of each reaction were performed, and data were calculated according to the $2^{-\Delta \Delta \mathrm{Ct}}$ method (Livak and Schmittgen, 2001).

Table 1. Full-length PhSERK cDNAs identified in Phalaenopsis.
\begin{tabular}{lccccc}
\hline Gene name & Full length (bp) & ORF (bp) & 5' UTR (bp) & 3' UTR (bp) & Protein length (aa) \\
\hline PhSERK1 & 2265 & 1899 & 245 & 121 & 633 \\
PhSERK2 & 2512 & 1887 & 256 & 369 & 629 \\
PhSERK3 & 2388 & 1848 & 283 & 257 & 616 \\
PhSERK4 & 2292 & 1848 & 101 & 343 & 616 \\
PhSERK5 & 2104 & 1869 & 51 & 184 & 623 \\
\hline
\end{tabular}

$\mathrm{ORF}=$ open reading frame; $\mathrm{UTR}=$ untranslated region.

\section{RESULTS}

\section{Identification of PhSERKs from Phalaenopsis orchid}

Partial PhSERK1 gene fragments from Phalaenopsis were obtained using degenerate primers designed from the conserved domain and the full-length sequence by RACE. Assembly of PCR fragments generated a full-length sequence of 2049 bp (Table 1). To identify additional SERK sequences in Phalaenopsis, we searched the Orchidstra database for potential cDNA clones. Four clones were found to be unique among 19 SERK sequences and were obtained by PCR with specific primers designed from SERK cDNA sequences (Orchidstra accession Nos. PATC125548, PAVEGC012987, PAALLC028299, and PATC125663). Fulllength PhSERK cDNA for these 4 ESTs were obtained using 5' and 3' RACE. All 5 PhSERKs contained both 5' and 3' untranslated regions (UTR) of 51 to $369 \mathrm{bp}$. Sequence information for the 5 cloned PhSERKs is shown in Table 1. The deduced length of proteins ranged from 616-633 amino acids.

Except for PhSERK3 and 4, the PhSERK proteins showed features of the protein kinase superfamily because they contained domains typical of SERK proteins found in other species, including signal peptide, leucine-zipper, and 5 LRR domains, as well as a serine-proline-proline motif, a hydrophobic transmembrane domain, and a Ser/Thr kinase domain comprising 11 sub-domains of conserved amino acid sequences (Figure 2). The signal peptides of PhSERK1, PhSERK2, and PhSERK5 contained a potential cleavage site between 24 and 29 amino acids as predicted by the SignalP 4.0 Server tool (http://www.cbs.dtu.dk/services/ SignalP/) (Figure S2). The Ser/Thr protein-kinase active-site signature was located from 319601 aa, the PhSERK transmembrane region was at 256-294 aa, and a cytoplasmic tail spanned 295-650 aa (Figure 2). PhSERK3 and 4 lacked the signal peptide, which was also observed for the DcSERK protein involved in plant embryogenesis (Schmidt et al., 1997).

\section{Phylogenetic relationships between Phalaenopsis SERK proteins}

Full-length amino acid sequences for the 5 PhSERKs and other SERK proteins were used for phylogenetic analysis. Compared to other SERK proteins from monocots and dicots, PhSEKK1 and 2 showed the highest similarity with coconut CnSERK and pineapple AcSERK1 and 2 as well as Cyrtochilum loxense CISERK, rice OsSEK1, alfalfa MtSERK1, maize ZmSERK1 and 2, Arabidopsis AtSERK1 and 2, and potato StSERK1 (Figure 3 and 
Table S2). PhSERK3 and 4 were grouped with the other SERK proteins lacking the signal peptide. PhSERK5 clustered with PpSERK1 and 2, with 64-65\% similarity for both proteins (Figure 3 and Table S2).

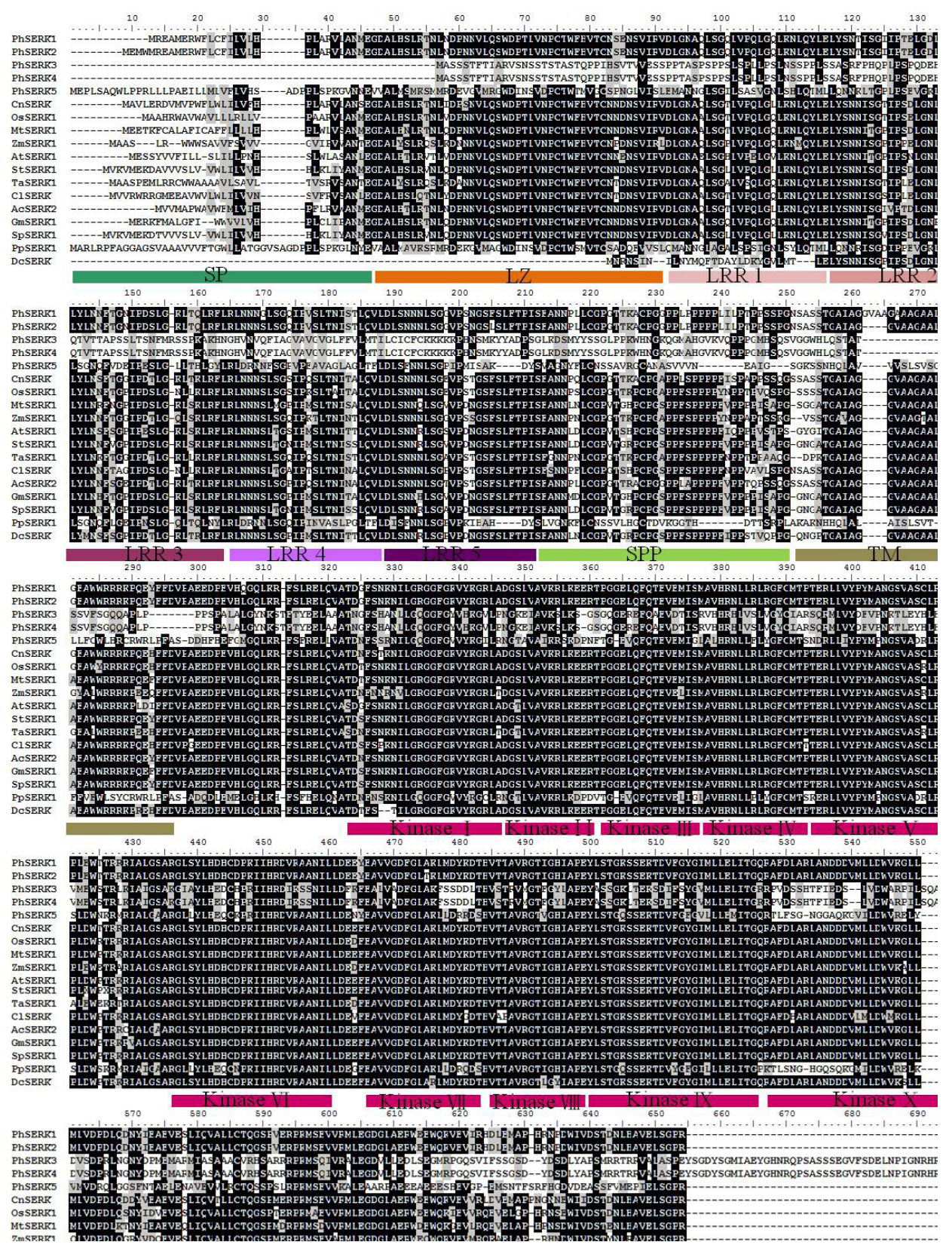

Figure 2. Alignment of predicted amino acid sequences of SERK family proteins. Identical amino acids are shaded in black. Different domains are indicated above the corresponding sequences. 


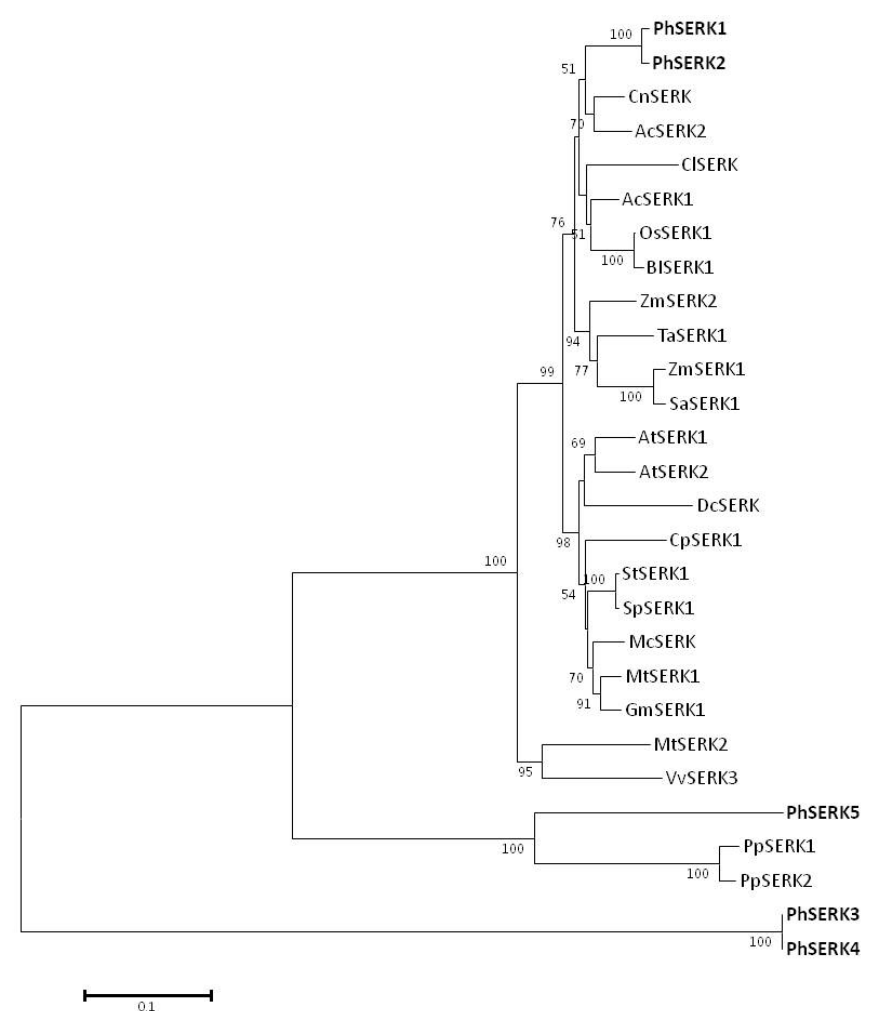

Figure 3. Phylogenetic tree of known somatic embryogenesis receptor-like kinases (SERKs) based on predicted full-length amino acid sequences. The 23 other plant SERKs and accession numbers in GenBank are CnSERK (AY791293.2), AcSERK1 (HM236375.2), AcSERK2 (HM236376.1), ClSERK (FN994192.1), OsSERK1 (AB188247.1), OsBISERK1 (AY463361.1), ZmSERK1 (NM_001111662.1), ZmSERK2 (NM_001111663.1), TaSERK1 (JF808017.1), SaSERK1 (GQ283907.1), AtSERK1 (NM_105841.4), AtSERK2 (NM_103144.4), DcSERK (U93048.1), CpSERK1 (EF672247.2), StSERK1 (EF175216.1), SpSERK1 (EF623824.1), McSERK (JX863894.3), MtSERK1 (AY162176.1), MtSERK2 (HM_640001.1), GmSERK1 (EU869193.1), VvSERK3 (XM_002262662.1), PpSERK1 (AJ841698.1), and PpSERK2 (AJ841697.1).

\section{Expression pattern of PhSERKs in Phalaenopsis tissue}

PhSERK transcripts were detected in all tissue examined, although at varying levels of expression. The expression pattern in SAM was similar among the 5 PhSERK genes and was higher than in root tips, leaf, and inflorescence (Figure 4). The expression in SAM was higher for PhSERK1 and 3 than the other PhSERKs (Figure 4). In floral buds, expression of all 5 PhSERKs was downregulated with development stage, was maintained at relatively low levels, and was greatly reduced in the stage 6 floral bud (FB6) of 0.8-0.99 cm. Expression of all PhSERKs was increased in the semi-open and fully open flower stage (FB7-8). The PhSERK4 transcript was most abundant in the first flower bud stage (FB1) and was maintained at relatively high levels in subsequent floral stages. In floral parts of open flowers, PhSERKs showed similar expression patterns, except the level was higher for PhSERK4 than other PhSERKs in pollinia (Figure 4, PL). 


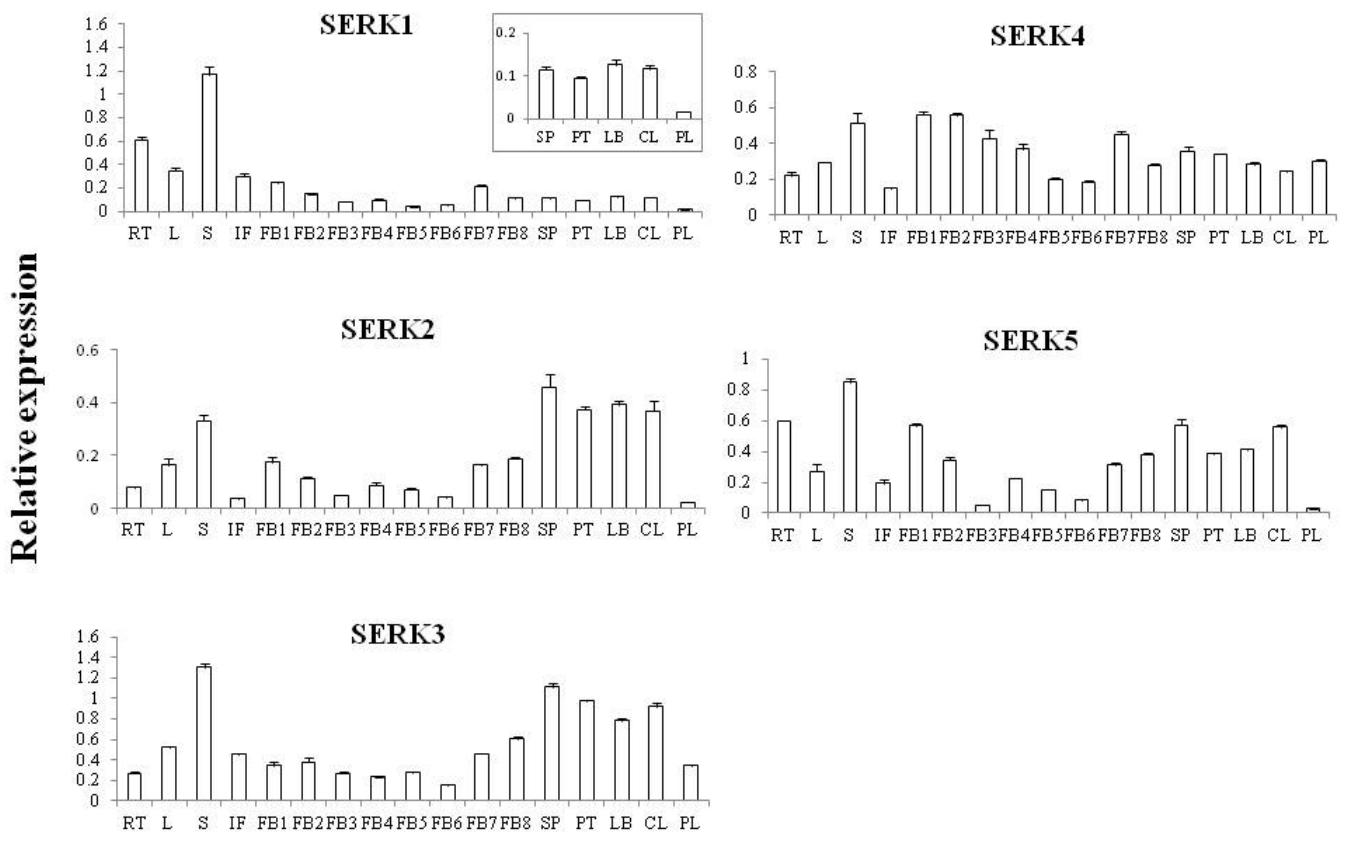

Figure 4. Real-time PCR of PhSERKs in different tissues and flower developmental stages of Phalaenopsis Sogo Vivien. Relative mRNA levels were normalized to that of the housekeeping gene PhActin. $\mathrm{RT}=$ root tip; $\mathrm{L}=$ leaf; $\mathrm{S}=$ shoot apical meristem; IF = inflorescence; $\mathrm{FB}=$ floral bud; FB1 $=0.20-0.29 \mathrm{~cm} ; \mathrm{FB} 2=0.30-0.39 \mathrm{~cm} ; \mathrm{FB} 3=$ $0.40-0.49 \mathrm{~cm} ; \mathrm{FB} 4=0.50-0.59 \mathrm{~cm} ; \mathrm{FB} 5=0.60-0.79 \mathrm{~cm} ; \mathrm{FB} 6=0.80-0.99 \mathrm{~cm} ; \mathrm{FB} 7=$ semi-open flower; FB8 = fully open flower; $\mathrm{SP}=$ sepal; $\mathrm{PT}=$ petal; $\mathrm{LB}=$ labellum; $\mathrm{CL}=$ column; $\mathrm{PL}=$ pollinia.

\section{Expression of PhSERKs during protocorm development and PLB formation}

All 5 PhSERK genes showed transcriptional activity during 8 weeks of seed germination with development into protocorms. Except for PhSERK4, most PhSERKs were upregulated during the first week of germination (Figure 5), when the embryo swelled (Figure 1, P1). At week 2, PhSERK2 expression was increased 2- to 3-fold as compared to the initial stage during protocorm development, with the embryo emerged from the seed coat (Figure 5, P2) The PhSERK4 transcript level reached a maximum during week 3, with approximately 3-fold higher expression than during week 2 (P3) and then decreased to half of the level in control dry seeds (Figure 5, P0) during the last 3 weeks (P4-P6). All PhSERK genes showed reduced expression during protocorm development after 4 weeks until the first leaf formed in the tip and the absorbing hairs became elongated in the base (Figure 5, P4-P6).

To investigate the association between PhSERK gene expression and secondary PLB formation, we analyzed sectioned primary PLB explants during 5 weeks of culture. The mRNA levels of PhSERK1 and 5 slightly increased by week 1 after PLBs were wounded (Figure 1 and 5, PLB1). Expression of PhSERK5 was present beginning in week 1, peaked at week 3 (PLB3), and decreased thereafter (PLB4). Additionally, upregulation of the 5 PhSERKs at week 2 (PLB2) coincided with active cell division on wounded primary PLBs at this time, showing an approximately 6-fold increase in the level of PhSERK2. After 4-5 weeks, the 
wounded primary PLB explants could regenerate more secondary PLBs, but the transcriptional activity of PhSERK2 to 5 decreased (Figure 1 and 5, PLB4-5). However, expression of PhSERK1 peaked 4 weeks after wounding (Figure 5, PLB4); at this time, many secondary PLBs became apparent and formed from epidermal layers proximal to the wound site (Figure 1, PLB4). The expression pattern of PhSERK3 and PhSERK4 were similar during PLB development. For example, the level of both was decreased at week 1 (Figure 5, PLB1), increased at week 3 (PLB3), and then decreased (PLB4-5).

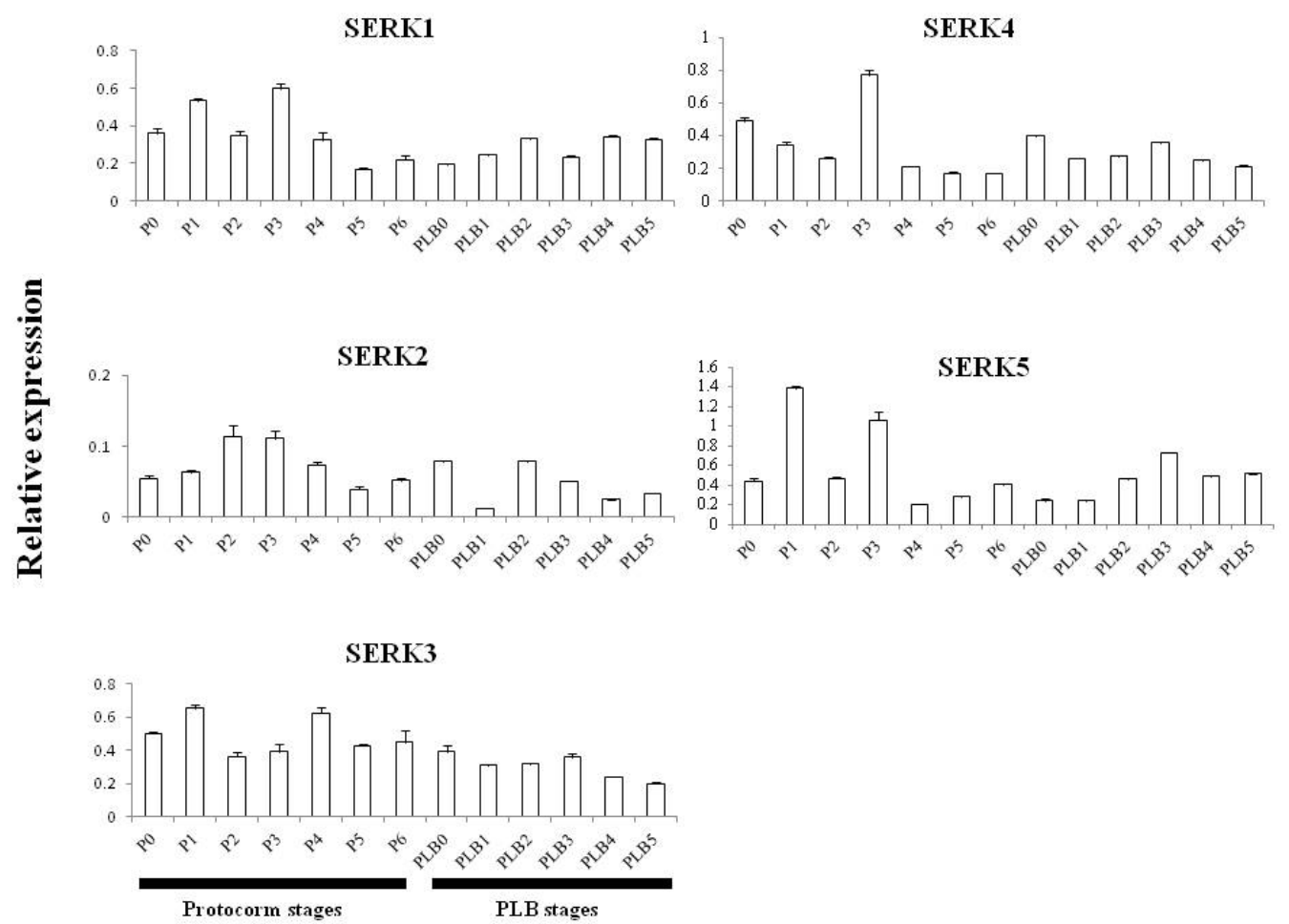

Figure 5. Expression of PhSERK genes during protocorm (P) and protocorm-like body (PLB) development in Phalaenopsis Sogo Vivien. Relative mRNA levels were normalized to that of PhActin. P0-P6 indicates weeks after seed germination. PLB0-PLB5 indicates culture time (weeks) on induction medium for the PLB explant after cutting out the apical part.

\section{DISCUSSION}

SERKs are thought to be important in several aspects of plant development in addition to somatic embryogenesis; these SERKs include AtSERK1 (Hecht et al., 2001), TcSERK (Santos et al., 2005), StSERK (Sharma et al., 2008), TaSERKs (Singla et al., 2008), GmSERK1 (Yang et al., 2011), and AcSERKs (Ma et al., 2012a,b). Somatic embryogenesis offers an advantage in plant propagation because many somatic embryos can be obtained in a short amount of time. Formation of PLBs in orchids is thought to be similar to somatic 
embryogenesis (Ishii et al., 1998; Mayer et al., 2010). Despite several studies of PLBs in Phalaenopsis (Young et al., 2000; Park et al., 2002), no studies have examined the link between SERK genes and PLB development in orchid. According to previous studies of other plants, SERKs are LRR-RLK factors and involved in somatic embryogenesis. We identified 5 PhSERK genes in the Phalaenopsis orchid. Three of these, PhSERK1,2, and 5, showed typical SERK protein structural features, as reported for other plant species. The high sequence similarity of PhSERK1 and 2 with CnSERK (Pérez-Núñez et al., 2009), AcSERK1 and 2 (Ma et al., 2012a,b), CISERK (Cueva et al., 2012), OsSERK (Ito et al., 2005), MtSERK1 (Nolan et al., 2003), ZmSERK1 (Baudino et al., 2001), and AtSERK1 (Hecht et al., 2001) suggests that PhSERK1 and 2 are involved in PLB formation or somatic embryogenesis, as has been proposed for orthologs from other species. Clustering of PhSERK5 with PpSERK1 and 2 (Figure 3) also points to a similar function in embryogenesis (Albrecht et al., 2005). However, PhSERK3 and 4 lack signal peptides and thus may have major functions in processes other than somatic embryogenesis (Figure S2). Signal peptide-lacking SERKs DcSERK (Schmidt et al., 1997) and TaSERK2 (Singla et al., 2008) were proposed to have a role in somatic embryogenesis. However, TaSERK3 showed the highest expression in developing seeds and appeared to be a brassinosteroid-associated kinase lacking a signal peptide, although it shares a characteristic C-terminal domain with other SERK proteins (Singla et al., 2008).

We analyzed the expression of the 5 PhSERK genes in different tissues, flower bud stages, seed germination, and PLB formation in Phalaenopsis orchids. Tissue-preferential expression of these PhSERKs in SAM was detected at high levels but at low levels in other tissues such as leaves, root tips, and inflorescence (Figure 4). The expression pattern of PhSERKs is reminiscent of their association with plant organ development as reported in other species. Moreover, AtSERK1 and 2 were reported to have redundant functions in sporophytic development and controlled male gametophyte production (Albrecht et al., 2005; Colcombet et al., 2005). Interestingly, PhSERK4 was preferentially expressed in pollinia as compared with other PhSERKs, and its expression peaked at the FB7 stage, when the anther was readily differentiated (Figure 4). PhSERK4 may have a role in sporophytic development.

Previous studies revealed that $S E R K$ transcripts are involved in both zygotic and somatic embryogenesis. Upregulation of PhSERKs, except for PhSER2, early during protocorm development, suggests that these PhSERKs are involved in morphogenesis of the Phalaenopsis orchid during seed germination. Schmidt et al. (1997) detected DcSERK mRNA only in early embryos up to the globular stage. PhSERK4 expression increased rapidly within 3 weeks of seed germination and decreased when seedlings were fully developed (Figure 5). This finding is consistent with those in PpSERKs (Albrecht et al., 2005) and GmSERK1 (Yang et al., 2011), which were also expressed in early plant embryogenesis, with decreased expression in later stages. Therefore, PhSERK4 appears to be a marker for normal protocorm development in this orchid.

Schellenbaum et al. (2008) observed that VvSERK1 and 3 were highly expressed during somatic embryo induction, before the visible appearance of embryos. VvSERK2 appears to be stably expressed and differs from the 2 other VvSERK genes (Schellenbaum et al., 2008). In wheat, TaSERK1 and 2 transcript levels were enhanced in embryogenic calli and may have a role during somatic embryogenesis, whereas TaSERK3 expression was the highest in developing seeds (Singla et al., 2008). StSERK1 was upregulated during the induction of somatic embryogenesis in potato and was continuously repressed during the embryo-transition phase (Sharma et al., 2008). CnSERK was detected in embryogenic tissues during somatic embryo- 
genesis, with expression greatly decreasing during somatic embryo development (PérezNúñez et al., 2009). When wounded explants were cultured in our PLB induction medium, PhSERK5 was highly expressed during the third week, when secondary PLBs became apparent (Figures 1 and 5). PhSERK5 transcription may be closely associated with the acquisition of embryogenic competence and cell proliferation during orchid PLB formation. Decreased levels of PhSERK2, 3, 4, and 5 during later stages of PLB formation suggest that they may be critical for PLB maturation (Figure 5).

In summary, the expression pattern observed of all 5 PhSERKs during PLB formation suggests their involvement during in vitro cellular differentiation. Our results agree well with those of previous studies of other species showing the expression of SERKs during early stage of somatic embryogenesis and detection until somatic embryo formation.

\section{Supplementary material}

\section{REFERENCES}

Albrecht C, Russinova E, Hecht V and Baaijens E (2005). The Arabidopsis thaliana somatic embryogenesis receptor-lke kinases 1 and 2 control male sporogenesis. Plant Cell 17: 3337-3349.

Baudino S, Hansen S, Brettschneider R, Hecht VF, et al. (2001). Molecular characterisation of two novel maize LRR receptor-like kinases, which belong to the SERK gene family. Planta 213: 1-10.

Boutilier K, Offringa R, Sharma VK, Kieft H, et al. (2002). Ectopic expression of BABY BOOM triggers a conversion from vegetative to embryonic growth. Plant Cell 14: 1737-1749.

Chang S, Puryear J and Cairney J (1993). A simple and efficient method for isolating RNA from pine trees. Plant Mol. Biol. Evol. 24: 1596-1599.

Chen YH, Tsai YJ, Huang JZ and Chen FC (2005). Transcription analysis of peloric mutants of Phalaenopsis orchids derived from tissue culture. Cell. Res. 15: 639-657.

Colcombet J, Boisson-Dernier A, Ros-Palau R, Vera CE, et al. (2005). Arabidopsis SOMATIC EMBRYOGENESIS RECEPTOR KINASES1 and 2 are essential for tapetum development and microspore maturation. Plant Cell 17: 3350-3361.

Cueva A, Concia L and Cella R (2012). Molecular characterization of a Cyrtochilum loxense somatic embryogenesis receptor-like kinase (SERK) gene expressed during somatic embryogenesis. Plant Cell Rep. 31: 1129-1139.

Gruszczyńska A and Rakoczy-Trojanowska M (2011). Expression analysis of somatic embryogenesis-related SERK, LEC1, VP1 and NiR ortologues in rye (Secale cereale L.). J. Appl. Genet. 52: 1-8.

Hecht V, Vielle-Calzada JP, Hartog MV, Schmidt ED, et al. (2001). The Arabidopsis SOMATIC EMBRYOGENESIS RECEPTOR KINASE 1 gene is expressed in developing ovules and embryos and enhances embryogenic competence in culture. Plant Physiol. 127: 803-816.

Hsu CC and Chen FC (2003). Plant regeneration from protocorm-like bodies induced in etiolated leaves of Phalaenopsis aphrodite Rchb. f. J. Chin. Soc. Hort. Sci. 49: 335-342.

$\mathrm{Hu} \mathrm{H}$, Xiong L and Yang Y (2005). Rice SERK1 gene positively regulates somatic embryogenesis of cultured cell and host defense response against fungal infection. Planta 222: 107-117.

Huang X, Lu XY, Zhao JT and Chen JK (2010). MaSERK1 gene expression associated with somatic embryogenic competence and disease resistance response in banana (Musa spp.). Plant Mol. Biol. Rep. 28: 309-316.

Ishii Y, Takamura T, Goi M and Tanaka M (1998). Callus induction and somatic embryogenesis of Phalaenopsis. Plant Cell Rep. 17: 446-450.

Ito Y, Takaya K and Kurata N (2005). Expression of SERK family receptor-like protein kinase genes in rice. Biochim. Biophys. Acta 1730: 253-258.

Ledwon A and Gaj MD (2009). LEAFY COTYLEDON2 gene expression and auxin treatment in relation to embryogenic capacity of Arabidopsis somatic cells. Plant Cell Rep. 28: 1677-1688.

Ledwon A and Gaj MD (2011). LEAFY COTYLEDON1, FUSCA3 expression and auxin treatment in relation to somatic embryogenesis induction in Arabidopsis. Plant Growth Regul. 65: 157-167.

Lin CC (1986). In vitro culture of flower stalk internodes of Phalaenopsis and Doritaenopsis. Lindleyana 3: 158-163.

Livak KJ and Schmittgen TD (2001). Analysis of relative gene expression data using real-time quantitative PCR and the 2(-Delta Delta C(T)) Method. Methods 25: 402-408. 
Ma J, He Y, Hu Z, Xu W, et al. (2012a). Characterization and expression analysis of AcSERK2, a somatic embryogenesis and stress resistance related gene in pineapple. Gene 500: 115-123.

Ma J, He YH, Wu CH and Liu HP (2012b). Cloning and molecular characterization of a SERK gene transcriptionally induced during somatic embryogenesis in Ananas comosus cv. Shenwan. Plant Mol. Biol. Rep. 30: 195-203.

Mayer JLS, Stancato GC and Appezzato-Da-Glória B (2010). Direct regeneration of protocorm-like bodies (PLBs) from leaf apices of Oncidium flexuosum Sims (Orchidaceae). Plant Cell Tiss. Org. Cult. 103: 411-416.

Nolan KE, Irwanto RR and Rose RJ (2003). Auxin upregulates MtSERK1 expression in both Medicago truncatula rootforming and embryogenic cultures. Plant Physiol. 133: 218-230.

Nolan KE, Kurdyukov S and Rose RJ (2009). Expression of the SOMATIC EMBRYOGENESIS RECEPTOR-LIKE KINASE1 (SERK1) gene is associated with developmental change in the life cycle of the model legume Medicago truncatula. J. Exp. Bot. 60: 1759-1771.

Park SY, Murthy HN and Paek KY (2002). Rapid propagation of Phalaenopsis from floral stalk-derived leaves. In Vitro Cell Dev. Biol. Plant 38: 168-172.

Park SY, Murthy HN and Paek KY (2003). Protocorm-like body induction and subsequent plant regeneration from root tip cultures of Doritaenopsis. Plant Sci. 164: 919-923.

Pérez-Núñez MT, Souza R, Sáenz L, Chan JL, et al. (2009). Detection of a SERK-like gene in coconut and analysis of its expression during the formation of embryogenic callus and somatic embryos. Plant Cell Rep. 28: 11-19.

Reisinger DM, Ball EA and Arditti J (1976). Clonal propagation of Phalaenopsis by means of flower-stalk node cultures. Jpn. Orchid Soc. Bull. 22: 9-14.

Santa-Catarina C, Hanai LR, Hanai LR and Viana AM (2004). SERK gene homolog expression, polyamines and amino acids associated with somatic embryogenic competence of Ocotea catharinensis Mez. (Lauraceae). Plant Cell Tiss. Org. Cult. 79: 53-61.

Santos MD, Romano E, Yotoko KSC and Tinoco MLP (2005). Characterisation of the cacao somatic embryogenesis receptor-like kinase (SERK) gene expressed during somatic embryogenesis. Plant Sci. 168: 723-729.

Santos MO, Romano E, Vieira LS, Baldoni AB, et al. (2009). Suppression of SERK gene expression affects fungus tolerance and somatic embryogenesis in transgenic lettuce. Plant Biol. 11: 83-89.

Savona M, Mattioli R, Nigro S, Falasca G, et al. (2012). Two SERK genes are markers of pluripotency in Cyclamen persicum Mill. J. Exp. Bot. 63: 471-488.

Schellenbaum P, Jacques A, Maillot P, Bertsch C, et al. (2008). Characterization of VvSERK1, VvSERK2, VvSERK3 and $V v L 1 L$ genes and their expression during somatic embryogenesis of grapevine (Vitis vinifera L.). Plant Cell Rep. 27: 1799-1809.

Schmidt ED, Guzzo F, Toonen MA and de Vries SC (1997). A leucine-rich repeat containing receptor-like kinase marks somatic plant cells competent to form embryos. Development 124: 2049-2062.

Sharma SK, Millam S, Hein I and Bryan GJ (2008). Cloning and molecular characterisation of a potato SERK gene transcriptionally induced during initiation of somatic embryogenesis. Planta 228: 319-330.

Shimada T, Hirabayashi T, Endo T and Fujii H (2005). Isolation and characterization of the somatic embryogenesis receptor-like kinase gene homologue (CitSERK1) from Citrus unshiu Marc. Sci. Hortic-Amsterdam 103: 233-238.

Shiu SH and Bleecker AB (2001). Plant receptor-like kinase gene family: diversity, function, and signaling. Sci. STKE 2001: re22.

Singla B, Khurana JP and Khurana P (2008). Characterization of three somatic embryogenesis receptor kinase genes from wheat, Triticum aestivum. Plant Cell Rep. 27: 833-843.

Somleva MN, Schmidt EDL and Vries SC (2000). Embryogenic cells in Dactylis glomerata L. (Poaceae) explants identified by cell tracking and by SERK expression. Plant Cell Rep. 19: 718-726.

$\mathrm{Su}$ YH, Zhao XY, Liu YB, Zhang CL, et al. (2009). Auxin-induced WUS expression is essential for embryonic stem cell renewal during somatic embryogenesis in Arabidopsis. Plant J. 59: 448-460.

Tamura K, Dudley J, Nei M and Kumar S (2007). MEGA4: Molecular evolutionary genetics analysis (MEGA) software version 4.0. Mol. Biol. Evol. 24: 1596-1599.

Thakare D, Tang W, Hill K and Perry SE (2008). The MADS-domain transcriptional regulator AGAMOUS-LIKE15 promotes somatic embryo development in Arabidopsis and soybean. Plant Physiol. 146: 1663-1672.

Thomas C, Meyer D, Himber C and Steinmetz A (2004). Spatial expression of a sunflower SERK gene during induction of somatic embryogenesis and shoot organogenesis. Plant Physiol. Biochem. 42: 35-42.

Tokuhara K and Mii M (1993). Micropropagation of Phalaenopsis and Doritaenopsis by culturing shoot tips of flower stalk buds. Plant Cell Rep. 13: 7-11.

Yang C, Zhao TJ, Yu DY and Gai JY (2011). Isolation and functional characterization of a SERK gene from soybean [Glycine max (L.) Merr.]. Plant Mol. Biol. Rep. 29: 334-344.

Young PS, Murthy HN and Yoeup PK (2000). Mass multiplication of protocorm-like bodies using bioreactor system and 
subsequent plant regeneration in Phalaenopsis. Plant Cell Tiss. Org. Cult. 63: 67-72.

Zakizadeh H, Stummann BM, Lutken H and Muller R (2010). Isolation and characterization of four somatic embryogenesis receptor-like kinase (RhSERK) genes from miniature potted rose (Rosa hybrida cv. Linda). Plant Cell Tiss. Org. Cult. 101: 331-338. 